\title{
Results of Treatment of Venous Thromboembolic Complications in a Multidisciplinary Surgical Hospital of the Republic of Sakha (Yakutia)
}

\author{
Mikhail M. Vinokurov, $\mathrm{PhD}, \mathrm{ScD}^{1^{*}}$; Anton A. Yakovlev, $\mathrm{PhD}^{2}$; Vasily P. Ignatiev ${ }^{2}$; Natalia I. \\ Douglas, $\mathrm{PhD}, \mathrm{ScD}^{1}$; Innokenty D. Ushnitsky, $\mathrm{PhD}, \mathrm{ScD}^{1}$; Gennady A. Palshin, $\mathrm{PhD}, \mathrm{ScD}^{1}$; \\ Stepan S. Pavlov ${ }^{1}$ \\ ${ }^{1}$ M. K. Ammosov North-Eastern Federal University \\ ${ }^{2}$ Republican Hospital No. 2-Center for Emergency Medical Care \\ Yakutsk, the Republic of Sakha (Yakutia), Russia
}

\begin{abstract}
The article describes the main clinical risk factors for development of VTEC and the results of treatment in the Republic of Sakha (Yakutia) (RS(Y)). The main risk factors for VTEC development were oncological diseases in 75 (25.5\%) patients (15 of them with newly diagnosed cancer and 60 patients on the background of chemotherapy) and trauma of the musculoskeletal system in $55(18.7 \%)$ patients. In the structure of DVT of the lower limbs, an occlusion was most often (38.2\%) found in the ilealfemoral segments. PE was detected in $21(7.1 \%)$ patients. According to the results of the study, conservative methods of DVT treatment slightly prevailed over surgical methods (59.7\% versus $40.2 \%$ ) Personalized anticoagulant therapy based on the results of pharmacogenetic testing for a specific patient increases the effectiveness and safety of treatment. Catheter-directed thrombolytic therapy for massive and submassive PE is the preferred method in the absence of contraindications for surgical hospitals. In patients with ascending varicotrombosis, phlebectomy combined with excision of thrombosed tributaries speeds up recovery and reduces the time until discharge from the hospital. (International Journal of Biomedicine. 2019;9(3):260-262.)
\end{abstract}

Key Words: deep vein thrombosis $\bullet$ pulmonary embolism • activated partial thromboplastin time $\bullet$ computerized tomography

\section{Abbreviations}

APTT, activated partial thromboplastin time; CT, computerized tomography; DVT, deep vein thrombosis; FFT, free-floating thrombus; IVC, inferior vena cava; PE, pulmonary embolism; VTEC, venous thromboembolic complications.

\section{Introduction}

Venous thromboembolism is a multifactorial clinical disorder provoked by clinical risk factors and acquired or inherited predispositions to thrombosis with incidences of 104-183 per 100,000 person-years in Caucasian patients. ${ }^{(1,2)}$ The incidences for PE and DVT alone range from 29 to 78 and 45 to 117 , per 100,000 person-years, respectively. ${ }^{(3)}$

*Corresponding author: Prof. Mikhail M. Vinokurov, PhD, ScD. M. K. Ammosov North-Eastern Federal University. Yakutsk, the Sakha Republic, Russia. E-mail:mmv_mi@rambler.ru
According to the Russian clinical guidelines for the diagnosis, treatment and prevention of VTEC, the basis of the treatment tactics is angioagulant therapy, which is recommended for all patients with DVT in the absence of contraindications, regardless of the possibility of surgical or endovascular intervention on the main veins. ${ }^{(4,5)}$ Despite the introduction into clinical practice of new methods and drugs to improve the accuracy of diagnosis and effectiveness of VTEC treatment, PE remains one of the leading causes of death in economically developed countries and the Russian Federation. ${ }^{(6-8)}$

The aim of the study was to improve the results of treatment of patients with VTEC in RS(Y). 


\section{Materials and Methods}

The research group included 293 patients with VTEC who were treated in the First Surgical Department of the Republican Hospital №2 (the Center for Emergency Medical Care) in the period 2016-2018. To determine the tactics of treatment, for all patients the following examinations were conducted: clinical examination, ECG, clinical tests, D-dimer, coagulogram, fibrogastroduodenoscopy, and color duplex scanning of deep veins of the lower limbs; if the information from duplex scanning was insufficient, we performed selective cavography. To confirm the diagnosis of PE, we performed echocardiography, X-ray computed tomography of the thoracic organs, and angiopulmonography. To identify blood flow disorders, rheological indicators of plasma were determined.

\section{Results}

In our study, the main risk factors for VTEC development were oncological diseases in $75(25.5 \%)$ patients (15 of them with newly diagnosed cancer and 60 patients on the background of chemotherapy) and trauma of the musculoskeletal system in 55 $(18.7 \%)$ patients. Long-term immobility (strokes, craniocerebral injuries) was found in $48(16.3 \%)$ patients and hormone therapy (replacement or contraceptive) in $20(6.8 \%)$ patients. In $30(10.2 \%)$ cases, VTEC were diagnosed during pregnancy. In $26(8.8 \%)$ observations, the reason for VTEC could not be determined. Various forms of coronary heart disease, complicated by chronic heart failure in the stage of decompensation, were revealed in 15 (5.1\%) patients and catheter associated jugular vein thrombosis in $5(1.7 \%)$ patients. Lower limb varicose disease was detected in $19(6.4 \%)$ patients (Fig.1).

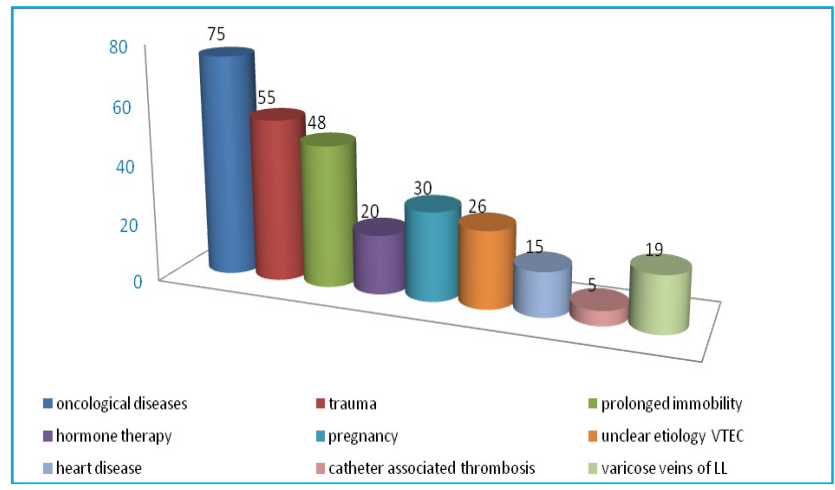

Figure 1.

In the structure of DVT of the lower limbs, an occlusion was most often (38.2\%) found in the ileal-femoral segments, and in $11(3.7 \%)$ cases the lesion was bilateral. Thrombotic lesions in the popliteal-femoral venous segment, in the popliteal tibia segment, and in the internal jugular vein were found in $80(27.3 \%)$, $50(17.0 \%)$ and $5(1.7 \%)$ cases, respectively; thrombosis of the infrarenal inferior vena cava with inferior vena cava syndrome was identified in $14(4.7 \%)$ cases. PE was detected in $21(7.1 \%)$ patients (Fig.2).

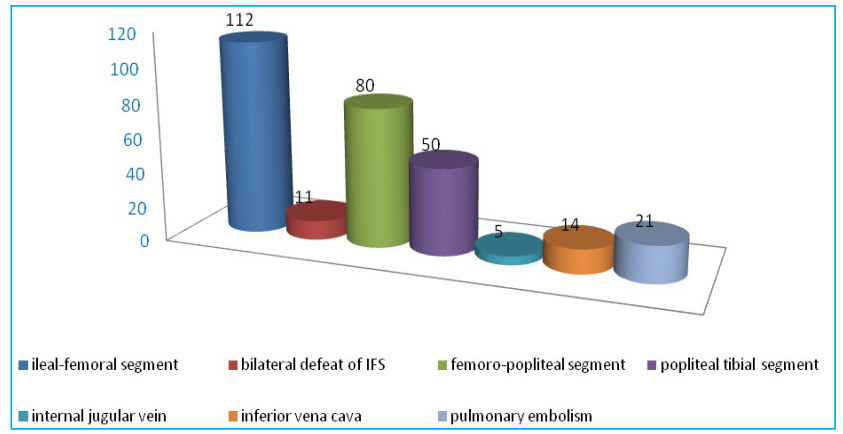

Figure 2.

A retrospective analysis showed that there was a significant increase in patients with VTEC during the research period: the number of patients was 87 in 2016, 99 in 2017, and 107 in 2018. Hospital mortality from PE in the study group of patients was $1.5 \%$ (5 cases) (Fig.3).

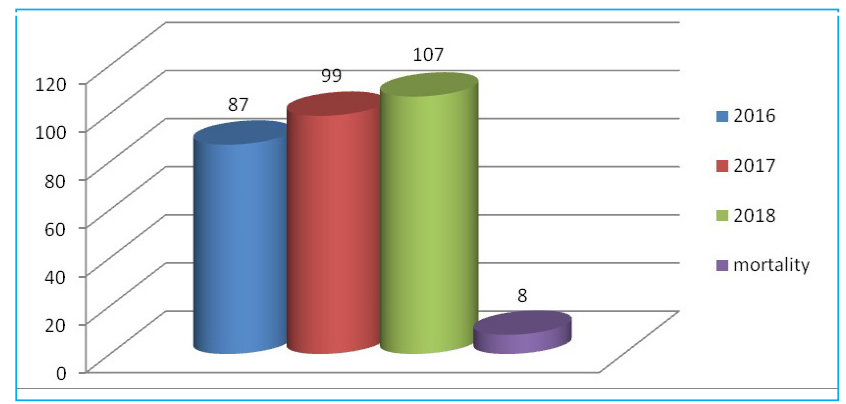

Figure 3.

The treatment tactics for patients with DVT of upper and lower limbs are identical. Starting therapy (parenteral administration of therapeutic doses of anticoagulants) begins with a reasonable suspicion of DVT development until the diagnosis is confirmed instrumental. We used the following anticoagulant therapy scheme for all patients: heparin was administered intravenously, 10,000 units at admission; further, depending on body weight 500 units $/ \mathrm{kg}$ per day were administered subcutaneously every 8 hours under the control of APTT, which, prior to the initiation of anticoagulant therapy, should be 1.5-2 times higher than normal values. Gastro-and hepatoprotectors were simultaneously prescribed for the period of treatment with anticoagulants. Further, on the seventh day, with the reduction heparin doses, we simultaneously administrated an oral anticoagulant for up to 2 days. Since 2017, when choosing oral anticoagulants we use personalized anticoagulant therapy based on the results of pharmacogenetic testing for a particular patient (Warfarin, Pradaxa, Xarelto), which increases the efficiency and safety of patient treatment. ${ }^{(7)}$ Reducing edema and softening of the muscles of the lower or upper extremities, as well as the initial or complete recanalization of thrombosed veins on ultrasound scans and improved coagulation by the end of 14 days of anticoagulant therapy, were regarded as positive results of anticoagulant therapy. The duration of oral anticoagulant intake on an outpatient basis can last from 3 to 6 months. It is also necessary to use lower extremity compression therapy 
In 10 cases, with the threat of detachment of FFT with a length of $4 \mathrm{~cm}$ or more, we used the installation of a permanent IVC filter. A temporary cava filter was installed in 71 patients and was removed 14-30 days after installation. A permanent IVC filter was installed in 27 cases, taking into account the severity of the underlying disease and the age of the patient. Numerous studies show encouraging results in the treatment of acute DVT with regional catheter thrombolysis. $(4,5,9)$ Satisfactory results of treatment are noted in $75 \%$ $90 \%$ of observations. ${ }^{(6,7,9)}$ In our research, after conducting regional catheter thrombolysis, the quality of life of patients was higher than in the group of patients who received only anticoagulant therapy. Catheter-directed thrombolytic therapy for massive and submassive PE was performed in 12 patients with positive dynamics of treatment. In cases of the ascending varicotromboses, active surgical tactics were applied in the volumes of crosssectomy ${ }^{(8)}$ or combined phlebectomy with excision of thrombosed tributaries in 35 patients. All patients were discharged with recovery on the seventh day after the surgery. According to the results of the study, conservative methods of DVT treatment slightly prevailed over surgical methods (59.7\% versus $40.2 \%$ ) (Fig. 4).

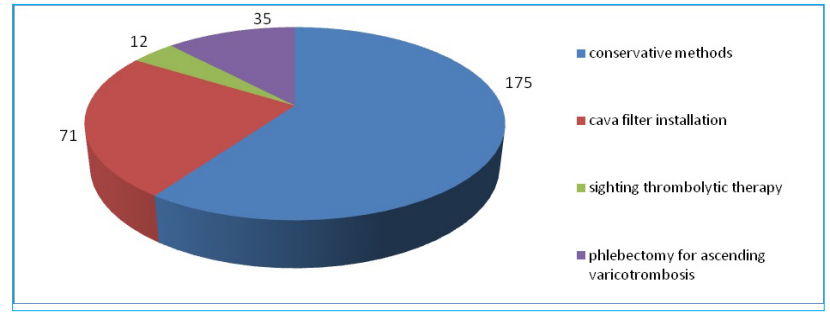

Figure 4.

\section{In conclusion:}

1. According to the results of this study, the leading factors contributing to the development of VTEC in RS(Y) are oncological diseases, trauma of the musculoskeletal system, prolonged immobility (strokes, craniocerebral injuries), pregnancy, contraception, and various forms of coronary heart disease.

2. A sharp increase in the number of patients with VTEC for the period 2016 - 2018 is recorded, which is seriously alarming.

3. The treatment tactic for DVT is based on anticoagulant therapy, which is indicated for all patients in the absence of contraindications, regardless of the possibility of surgical or endovascular intervention on the main veins.

4. Personalized anticoagulant therapy based on the results of pharmacogenetic testing for a specific patient increases the effectiveness and safety of treatment.
5. The installation of a permanent IVC filter in the threat of detachment of FFT with a length of $4 \mathrm{~cm}$ or more remains relevant in surgical hospitals.

6. Catheter-directed thrombolytic therapy for massive and submassive PE is the preferred method in the absence of contraindications for surgical hospitals.

7. In patients with ascending varicotrombosis, phlebectomy combined with excision of thrombosed tributaries speeds up recovery and reduces the time until discharge from the hospital.

\section{Competing Interests}

The authors declare that they have no competing interests.

\section{References}

1.Heit JA. Epidemiology of venous thromboembolism. Nat Rev Cardiol. 2015;12(8):464-74. doi: 10.1038/nrcardio.2015.83.

2.Hansson PO, Welin L, Tibblin G, Eriksson H.Deep vein thrombosis and pulmonary embolism in the general population. 'The Study of Men Born in 1913'. Arch Intern Med. 1997;157(15):1665-70.

3. Cushman M, Tsai AW, White RH, Heckbert SR, Rosamond WD, Enright P, Folsom AR. Deep vein thrombosis and pulmonary embolism in two cohorts: the longitudinal investigation of thromboembolism etiology. Am J Med. 2004;117(1):19-25.

4.Savelyev VS, Chazov EI, Gusev EI, Kirienko AI, Gelfand BR, Piradov MA, et al. Russian clinical guidelines for the diagnosis, treatment and prevention of venous thromboembolic complications. Flebologiya. 2010;4(2):1-37.[Article in Russian].

5.Russian clinical guidelines "Diagnosis, treatment and prevention of venous thromboembolic complications". Edited by A.I. Kirienko. Moscow; 2015. [In Russian].

6.Kalinin RE, Suchkov IA, Pshennikov AS, Agapov AB. Quality of life of patients with venous thromboses at different variants of anticoagulant therapy. Angiology and Vascular Surgery. 2016;22(2):15-20. [Article in Russian].

7.Cascorbi I. The promises of personalized medicine. Eur J Clin Pharmacol. 2010 Aug;66(8):749-54. doi: 10.1007/ s00228-010-0858-6.

8.Eriksson BI, Dahl OE, Huo MH, Kurth AA, Hantel S, Hermansson K, et al.; RE-NOVATE II Study Group. Oral dabigatran versus enoxaparin for thromboprophylaxis after primary total hip arthroplasty (RE-NOVATE II*). A randomized, double-blind, non-inferiority trial. Thromb Haemost. 2011 Apr;105(4):721-9. doi: 10.1160/TH10-10-0679.

9. Heit JA, Silverstein MD, Mohr DN, Petterson TM, O'Fallon WM, Melton LJ 3rd. Predictors of survival after deep vein thrombosis and pulmonary embolism: a population-based, cohort study. Arch Intern Med. 1999;159(5):445-53. 